Pesq. Vet. Bras. 37(7):741-748, julho 2017

DOI: $10.1590 / \mathrm{S} 0100-736 \mathrm{X} 2017000700015$

\title{
Infestação por carrapatos Argasidae e Ixodidae em pequenos mamíferos silvestres da Estação Experimental Rafael Fernandes, Mossoró/RN ${ }^{1}$
}

\author{
Josivania S. Pereira ${ }^{2 *}$, Thiago F. Martins ${ }^{3}$, Sebastián Muñoz-Leal ${ }^{3}$, Marcos G. \\ Lopes $^{3}$, Marcelo B. Labruna ${ }^{3}$, Kaliane A.R. de Paiva², Moacir F. de Oliveira ${ }^{2}$ \\ e Sílvia M.M. Ahid ${ }^{2}$
}

\begin{abstract}
Pereira J.S., Martins T.F., Muñoz-Leal S., Lopes M.G., Labruna M.B., Paiva K.A.R., Oliveira M.F. \& Ahid S.M.M. 2017. [Infestation by ticks Argasidae and Ixodidae on small wild mammals at the Experimental Station Rafael Fernandes, Mossoró/RN, Brazil.] Infestação por carrapatos Argasidae e Ixodidae em pequenos mamíferos silvestres da Estação Experimental Rafael Fernandes, Mossoró/RN. Pesquisa Veterinária Brasileira 37(7):741-748. Universidade Federal Rural do Semi-Árido, Av. Francisco Mota 572, Bairro Costa e Silva, Mossoró, RN 59625-900, Brazil. E-mail: josigej@ufersa.edu.br

Few studies have assessed the diversity of ectoparasites and their associated hosts occurring within the Caatinga biome in northeastern Brazil. Considering this lack of knowledge, in this study we aimed to identify and determine the occurrence of ticks collected from small mammals at the Estação Experimental Rafael Fernandes, in Rio Grande do Norte state, Brazil. From January 2014 to February 2015, we captured 52 marsupials (38 Gracilinanus agilis and 14 Monodelphis domestica) and 10 rodents (5 Wiedomys sp., 4 Thrichomys sp. and 1 Rattus norvegicus). We identified the ticks Amblyomma auricularium, Amblyomma parvum, Amblyomma sp., Ornithodoros mimon and Ornithodoros sp. by a morphological study, the use of taxonomic keys, and the partial sequencing of the tick mitochondrial $16 \mathrm{~S}$ rDNA gene. All the tick-host associations found in this study are reported for the first time in Rio Grande do Norte and constitute new ecological data concerning ectoparasites of small mammals in northeastern Brazil.

INDEX TERMS: Ticks, Argasidae, Ixodidae, wild mammals, Amblyomma auricularium, Amblyomma parvum, Gracilinanus agilis, Monodelphis domestica, Ornithodoros mimon.
\end{abstract}

RESUMO.- Poucos estudos avaliaram a diversidade de ectoparasitos e a associação deles com seus hospedeiros que ocorrem no bioma Caatinga, Nordeste do Brasil. Considerando-se essa falta de conhecimento, este estudo objetivou identificar e determinar a ocorrência de carrapatos coletados de pequenos mamíferos da Estação Experimental Rafael Fernandes, no Rio Grande do Norte, Brasil. De janeiro de 2014 a fevereiro de 2015 foram capturados 52 marsupiais (38 Gracilinanus agilis e 14 Monodelphis domestica)

\footnotetext{
${ }^{1}$ Recebido em 15 de junho de 2015.

Aceito para publicação em13 de abril de 2017.

${ }^{2}$ Centro de Ciências Biológicas e da Saúde (CCBS), Universidade Federal Rural do Semi-Árido (UFERSA), Av. Francisco Mota 572, Bairro Costa e Silva, Mossoró, RN 59625-900, Brasil. *Autor para correspondência: josigej@ ufersa.edu.br

${ }^{3}$ Departamento de Medicina Veterinária Preventiva e Saúde Animal, Faculdade de Medicina Veterinária e Zootecnia, Universidade de São Paulo (USP), Av. Prof. Orlando Marques de Paiva 87, São Paulo, SP 05508-270, Brasil.
}

e 10 roedores (5 Wiedomys sp., 4 Thrichomys sp. e 1 Rattus norvegicus). Foram identificados os carrapatos Amblyomma auricularium, Amblyomma parvum, Amblyomma sp., Ornithodoros mimon e Ornithodoros sp., empregando estudo morfológico, chaves taxonômicas e sequenciamento parcial do gene mitocondrial $16 \mathrm{~S}$ rDNA de carrapatos. Todas as associações carrapato-hospedeiro encontradas neste estudo são relatadas pela primeira vez no Rio Grande do Norte e constituem novos dados ecológicos aplicáveis aos ectoparasitos de pequenos mamíferos no nordeste do Brasil.

TERMOS DE INDEXAÇÃO: Carapatos, Argasidae, Ixodidae, mamíferos silvestres, Amblyomma auricularium, Amblyomma parvum, carrapatos, Gracilinanus agilis, Monodelphis domestica, Ornithodoros mimon.

\section{INTRODUÇÃO}

A fauna silvestre brasileira é bastante diversificada e particularmente rica em espécies de mamíferos, contudo pouco se sabe sobre a epidemiologia e agentes patogênicos que 
acometem estes animais (Soares 2013). Ela pode representar uma valiosa fonte de informações, principalmente em localidades nunca antes estudadas, pois funciona como indicadora da qualidade do ambiente ao demonstrar, para agentes que hospeda, os desequilíbrios a que está exposta. Fato este pode sugerir boa capacidade de suporte e qualidade ambiental quando suas espécies sobrevivem bem (Thompson et al. 2009, Soares et al. 2015).

No Brasil, especificamente na região Nordeste, devido às políticas públicas de manejo dos ecossistemas, aliada a péssima distribuição de renda dos trabalhadores, principalmente os rurais, observa-se que a caça de animais silvestres tem funcionado como alternativa de sobrevivência econômica extra, pois permite a comercialização de peles para confecção de vestimentas e acessórios, além da produção de remédios. Este fato ocasiona a diminuição destes animais em seu habitat levando-os a sua extinção (Freitas 2012).

Dentre estes animais, os pequenos mamíferos das ordens Rodentia e Didelphimorphia apresentam importância ecológica e epidemiológica por serem presas e predadores nas cadeias ecológicas; realizarem dispersão de sementes e constituírem hospedeiros de ecto e endoparasitos, além de reservatórios de muitos agentes patogênicos (Reis et al. 2006, Cáceres 2012).

Quando em ambiente natural, podem ser acometidos por ectoparasitos com importância epidemiológica, já que estes organismos são transmissores de patógenos desencadeadores de doenças para animais e humanos, incluindo os Acari Ixodidae e Argasidae. Estes têm hábitos hematófagos, alimentando-se de uma grande variedade de vertebrados terrestres, incluindo seres humanos, animais domésticos e silvestres. Ainda podem ocasionar a estes últimos, doenças parasitárias que representam uma séria ameaça a sua população, uma vez que diminui a abundância dos mesmos em seu habitat (Mastropaolo et al. 2014, Sponchiado et al. 2015).

A fauna de carrapatos no Brasil compreende 68 espécies conhecidas, sendo que 46 pertencem à família Ixodidae (Dantas-Torres et al. 2009, Martins et al. 2014, Nava et al. 2014, Krawczak et al. 2015) e 22 à família Argasidae (Barros-Battesti et al. 2015, Wolf et al. 2016). A ocorrência destes carrapatos em animais silvestres tem sido reportada nos diferentes biomas brasileiros (Aragão 1936, Labruna et al. 2005, Szabó et al. 2009), porém na Caatinga, uma região de grande diversidade de fauna e flora, a fauna de carrapatos tem sido pouco estudada.

Considerando-se a importância epidemiológica dos pequenos mamíferos, unida escassez de dados na literatura sobre a ectofauna que os parasita no Rio Grande do Norte, objetivou-se identificar os parasitos Ixodidae e Argasidae nos pequenos mamíferos capturados em uma área de preservação natural da Estação Experimental Rafael Fernandes da Universidade Federal Rural do Semi-Árido (UFERSA), bem como determinar a ocorrência do parasitismo nesses hospedeiros.

\section{MATERIAL E MÉTODOS}

Animais experimentais e caracterização da localidade das amostragens. De janeiro de 2014 a fevereiro de 2015, capturaram-se espécimes machos e fêmeas não gestantes, de roedores e marsupiais silvestres de um fragmento de 26 hectares da Estação Experimental Rafael Fernandes ( $5^{\circ} 03^{\prime} 40^{\prime \prime} S$; 37 $23^{\circ} 51^{\prime \prime} \mathrm{W}$ e altitude de 72m), a qual apresenta clima quente e seco (Silva et al. 2012), está situada no distrito de Alagoinha, município de Mossoró/RN e é pertencente a UFERSA. Todos os procedimentos foram executados conforme as recomendações propostas pelo Sistema de Autorização e Informação em Biodiversidade (SISBIO, aprovação 43526-1), e as normas propostas pelo Comitê de Ética da UFERSA no uso de animais em pesquisa (Parecer no. 21/2013, processo no. 23091.00). As espécies de roedores e marsupiais foram identificadas por meio da morfologia, com base em literatura especializada (Reis et al. 2006, Bonvicino et al. 2008, Cáceres 2012).

Contenção e marcação dos animais. Utilizou-se dose de$120 \mathrm{mg} / \mathrm{kg}$ de quetamina (concentração de $1 \mathrm{~g} / 10 \mathrm{~mL}$ ) associada a $12 \mathrm{mg} / \mathrm{kg}$ de xilasina (concentração de $2 \mathrm{~g} / 100 \mathrm{~mL}$ ), administrada via intramuscular. Para evitar as recapturas, os animais foram identificados com brincos numerados (№ 01, medidas de $07 \mathrm{MM}$, com 3N) (Almeida et al. 2013).

Sessões de captura e armadilhas. Foram definidas 100 estações de captura, distribuídas em seis transectos distanciados $20 \mathrm{~m}$ entre si. Em cada estação foi colocada uma armadilha Tomahawk $(45 \times 17,5 \times 15,0 \mathrm{~cm})$ ou Sherman $(31 \times 8 \times 9 \mathrm{~cm})$, de forma alternada e no solo, com distância de $20 \mathrm{~m}$ entre armadilhas. Em cada mês de coleta, as sessões de captura foram realizadas durante seis noites consecutivas (Szabó et al. 2013). Como isca foi usada uma mistura feita com pasta de amendoim, sardinha, flocos de milho e banana (Almeida et al. 2013), distribuída antes do ocaso do sol (16 horas) e recolhida no início da manhã (5:30 horas) (Kim et al. 2010).

Coleta dos ectoparasitos e soltura dos animais. Realizou-se uma inspeção por toda superfície corpórea e quando observados carrapatos, estes foram removidos individualmente por giro sobre seu eixo a fim de evitar a perda de estruturas taxonômicas (Pereira et al. 2012). Após coleta dos ectoparasitos, os animais foram soltos no local exato de sua captura.

Identificação dos ectoparasitos. As ninfas de carrapatos foram identificadas no Laboratório de Parasitologia Animal da UFERSA com o auxílio de chave dicotômica e pictórica segundo Martins et al. (2010). Quando foi possível a classificação morfológica dos estágios larvais, utilizaram-se chaves taxonômicas de Jones \& Clifford (1972), Kohls et al. (1969) e Barros-Battesti et al. (2013). Foram realizadas morfometria e quetotaxia em larvas previamente submetidas à clarificação em solução de hidróxido de potássio $10 \%$ e montadas em meio de Hoyer. As larvas foram mensuradas por fotografias digitais capturadas com uma câmera Olympus DP70 acoplada a um microscópio Olympus BX40, usando o software Image-Plus Pro v5.1 e considerando os caracteres morfológicos propostos por Venzal et al. (2008). Quando não era possível à identificação através da morfologia, as larvas foram submetidas à extração de DNA para caracterização molecular (Mangold et al. 1998).

Análises moleculares. Os carrapatos foram previamente lavados com etanol a $70 \%$ e secos ao ar durante 20 minutos em papel estéril. Cada carrapato foi colocado em um microtubo estéril de 1,5 $\mathrm{ml}$, macerado com agulha 40x12 e processado com o Kit Wizard ${ }^{\circledR}$ Genomic DNA Purification (Promega corporation, Madison, WI 53711-5399, EUA) de acordo com as instruções do fabricante com modificações. A extração de DNA de algumas amostras de larvas a partir do mesmo hospedeiro foi processada em pools de até no máximo três indivíduos. Realizou-se uma PCR usando os primers 16S+ (forward, 5'-CCGGTCTGAACTCAGATCAAGT-3') e 16S- (reverse, 5'- GCTCAATGATTTTTTAAATTGCTGT-3'), que amplificam um fragmento de $\approx 460$-pb do gene mitocondrial $16 \mathrm{~S}$ rRNA do carrapato (Mangold et al. 1998). Todos os produtos da PCR (5 $\mu \mathrm{L}$ DNA amplificado acrescido de $2 \mu \mathrm{l}$ de corante Gel Loading Buffer (Invi- 
trogen ${ }^{\circledR}$ Carlsbad, CA) foram submetidos à eletroforese horizontal em gel de agarose $1,5 \%$ acrescido de $\mathrm{SYBR}^{\circledR}$ Safe DNA Gel Stain $(0,1 \mu \mathrm{l} / \mathrm{mL})$ e tampão de corrida TBE $0,5 \mathrm{X}$ pH 8,0 à $110 \mathrm{~V} / 50 \mathrm{~mA}$. Visualizou-se o gel em luz ultravioleta (UV) em câmara escura (Alphalmager ${ }^{\circledR}$ ). As amostras que revelaram bandas de DNA na altura do controle positivo foram consideradas positivas. Como controle positivo utilizou-se DNA de Ixodes uriae White 1852 e como controle negativo, água Mili-Q autoclavada. Os produtos da PCR foram purificados através do produto comercial ExoSAP-IT (Amersham Biosciences, Piscataway, NJ), adicionando-se $4 \mu \mathrm{L}$ do reagente e $10 \mu \mathrm{L}$ da amostra amplificada na PCR. Os produtos purificados foram submetidos ao sequenciamento através do uso de Kit comercial BigDye ${ }^{\circledR}$ Terminator v 3.1 (Applied Biosystems/Austin, TX) de acordo com o fabricante e logo sequenciados em um sequenciador automático (Applied Biosystems/Perkin Elmer, modelo ABI Prism 310 Genetic, FosterCity, CA). As sequências obtidas foram editadas através do programa Geneious R9 (Biomatter, New Zealand) e submetidas a análise de similaridade através do programa Basic Local Alignment Search Tool (BLAST) para verificar homologia com sequências correspondentes disponíveis na base de dados GenBank
(Altschul et al. 1990). Todas as análises moleculares foram realizadas no Laboratório de Doenças Parasitárias (LDP) e Laboratório de Biologia Molecular Aplicado a Sorologia (LABMAS) da Universidade de São Paulo (USP).

Análise dos dados. Foram expressos em média, desvio padrão, valores mínimos, máximos e coeficiente de variação (CV\%), calculados pelo programa estatístico SPSS (Statistical Package for the Social Sciences) 21.0. A ocorrência dos carrapatos foi dada pelo número de carrapatos dividido pelos animais capturados.

\section{RESULTADOS}

Foram capturados 62 pequenos mamíferos infestados por carrapato no pavilhão auricular, na região ocular e nos membros. Foram identificados os hospedeiros Gracilinanus agilis (Burmeister, 1854), Wiedomys (Hershkovitz, 1959), Monodelphis domestica (Wagner, 1842), Thrichomys (Trouessart, 1880) e Rattus norvegicus (Berkenhout, 1769) (Fig.1). Foram coletadas duas espécies de Ixodidae e uma de Argasidae (Quadro 1).
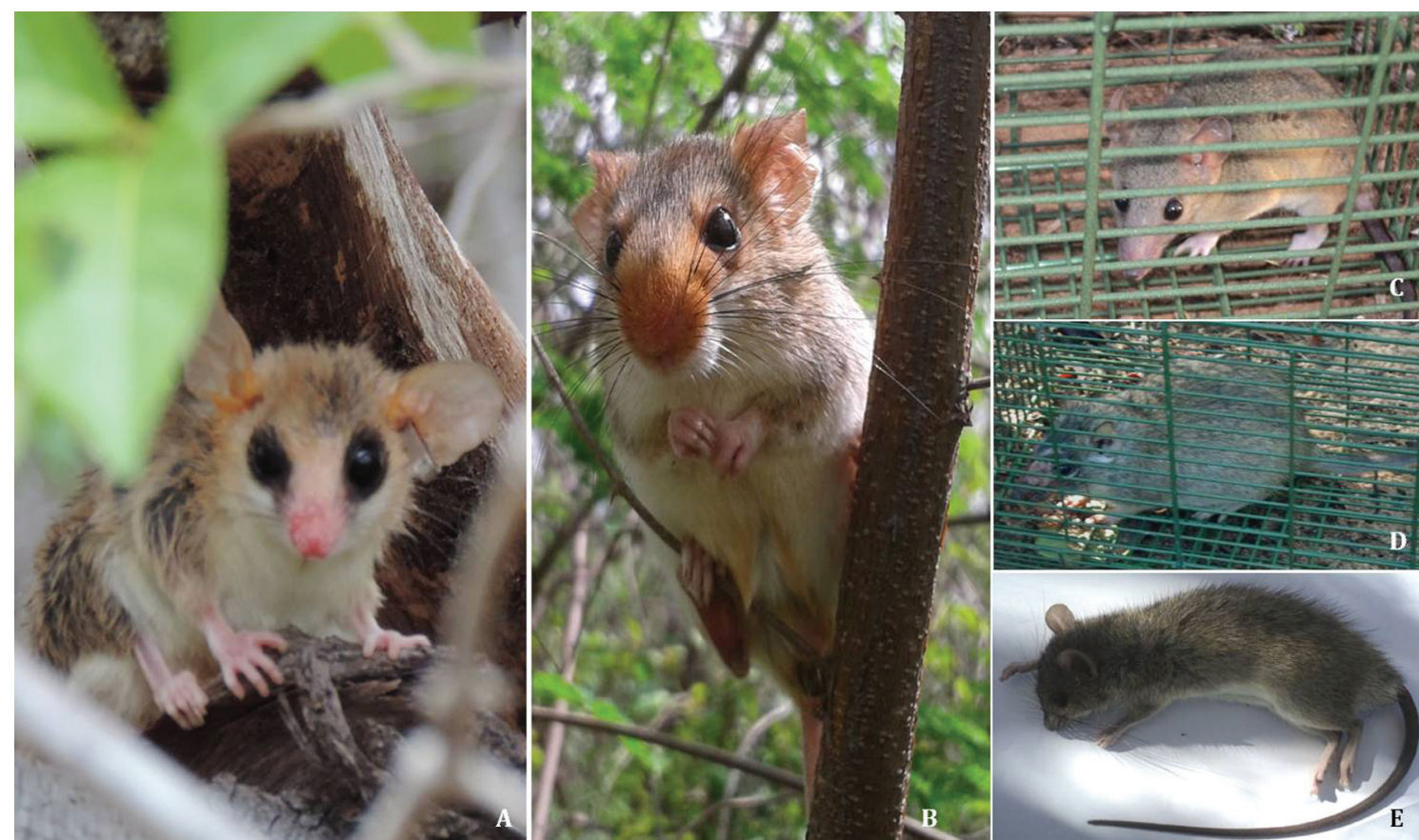

Fig.1. Hospedeiros capturados na Estação Experimental Rafael Fernandes, Rio Grande do Norte, Brasil. (A) Gracilinanus agilis. (B) Wiedomys sp. Captura em armadilha Tomahawk: (C) Monodelphis domestica. (D) Thrichomys sp. (E) Rattus norvegicus após anestesia.

Quadro 1. Carrapatos coletados de pequenos mamíferos capturados na Estação Experimental Rafael Fernandes, Rio Grande do Norte, Brasil, de janeiro de 2014 a fevereiro de 2015

\begin{tabular}{lcccc}
\hline Hospedeiro (n) & $\begin{array}{c}\text { Hospedeiros } \\
\text { parasitados }\end{array}$ & $\begin{array}{c}\text { Espécie de } \\
\text { Carrapato }\end{array}$ & $\begin{array}{c}\text { o de larva e ninfa coletado } \\
\text { no hospedeiro }\end{array}$ & $\begin{array}{c}\text { No total de ectopa- } \\
\text { rasitos coletado }\end{array}$ \\
\hline Monodelphis & 11 & A. auricularium & $168 \mathrm{~L} ;$ 6N & 174 \\
domestica (14) & 1 & Ornithodoros sp. & $1 \mathrm{~L}$ & 1 \\
& 1 & O. mimon & $1 \mathrm{~L}$ & 1 \\
Gracilinanus agilis (38) & 10 & A. auricularium & $11 \mathrm{~L}$ & 11 \\
& 28 & O. mimon & $40 \mathrm{~L}$ & 40 \\
Wiedomys sp.(5) & 5 & Amblyomma sp. & $12 \mathrm{~L}$ & 12 \\
Rattus norvegicus (1) & 1 & Amblyomma sp. & $6 \mathrm{~L}$ & 6 \\
Thrichomys sp. (4) & 1 & A. auricularium & $10 \mathrm{~L} ; 21 \mathrm{~N}$ & 31 \\
& 1 & A. parvum & $15 \mathrm{~N}$ & 15 \\
& 1 & Ornithodoros sp. & $1 \mathrm{~L}$ & 1
\end{tabular}

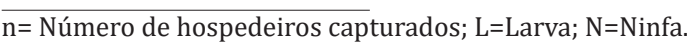




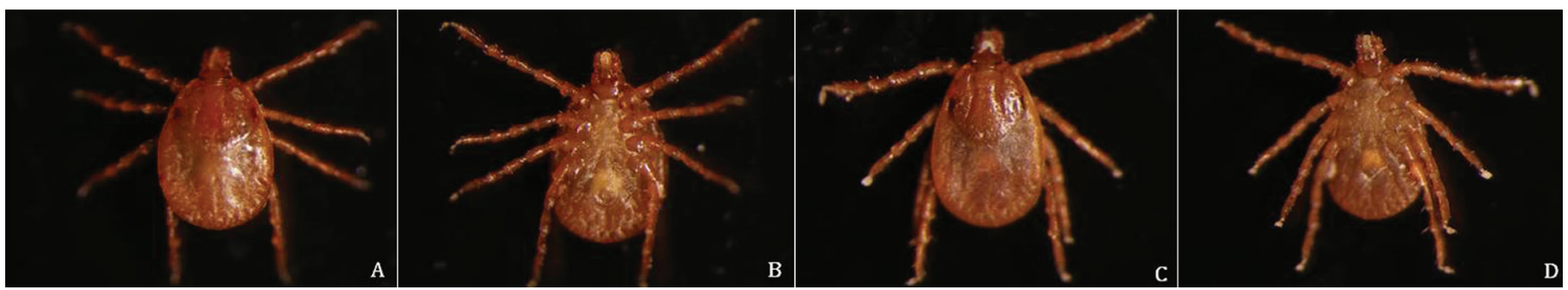

Fig.2. Ninfa de Amblyomma auricularium. Obj.5.6x: (A) Vista dorsal. (B) Vista ventral. Ninfa de Amblyomma parvum: (A) Vista dorsal. (B) Vista ventral.

Um total de 12 e 6 larvas de carrapato coletadas de Wiedomys sp. e $R$. norvegicus, respectivamente, foram classificadas somente até o gênero Amblyomma (Quadro 1). As ninfas obtidas dos marsupiais e roedores foram identificadas como Amblyomma auricularium (Conil, 1878) e Amblyomma parvum (Aragão, 1908) (Fig.2).

A análise morfométrica das características (Quadro 2) da larva de Argasidae coletada de Thrichomys sp., permitiu a sua identificação como Ornithodoros mimon (Kohls et al. 1969) (Fig.3). A mesma classificação foi dada as larvas obtidas de G. agilis (Quadro 3). Estas apresentaram como características: estado de alimentação ingurgitada, cápsula do órgão de Haller reticulada, placa dorsal em forma de pera, 14 pares de cerdas dorsais (11 dorsolaterais, 3 centrais), 7 pares ventrais mais 1 par anal. Nos palpos, no artículo I nenhuma seta foi observada, enquanto que nos artículos II, III e IV encontraram-se 4, 5 e 9 setas, respectivamente. 0 hipostômio apresentou um ápice arredondado, 15 dentes na fila um, 12 na dois, 5 na três e 3 na quatro. A fórmula dental apical, medial e basal foi respectivamente de 4.4/3.3, 2.2 e 2.2.

Uma larva de Ornithodoros sp. coletada em M. domesti-

\section{Quadro 2. Morfometria da larva de Ornithodoros mimon coletada de Thrichomys sp. capturado na Estação Experimental Rafael Fernandes, RN, Brasil}

\begin{tabular}{|c|c|}
\hline Características & Valores $(\mu \mathrm{m})$ \\
\hline Estado de alimentação & Ingurgitada \\
\hline Placa dorsal: comprimento & 166,78 \\
\hline Cerda dorsal: total de pares & 14 \\
\hline Cerda dorsal: total de pares dorsolaterais & 11 \\
\hline Cerda dorsal: pares central & 3 \\
\hline Cerda ventral: total de pares & $7+1 \mathrm{Pms}$ \\
\hline Placa dorsal: largura & 130,65 \\
\hline $\begin{array}{l}\text { Comprimento da base do capítulo } \\
\text { até a inserção do hipostômio }\end{array}$ & 158,38 \\
\hline Comprimento base do capítulo & 133,68 \\
\hline Largura do capítulo & 184,10 \\
\hline Comprimento do palpo & 210,49 \\
\hline Comprimento do artículo I & 202,65 \\
\hline Comprimento do artículo II & 52,11 \\
\hline Comprimento do artículo III & 71,92 \\
\hline Comprimento do artículo IV & 60,67 \\
\hline Largura do artículo I & 41,20 \\
\hline Largura do artículo II & 34,32 \\
\hline Largura do artículo III & 40,65 \\
\hline Largura do artículo IV & 35,43 \\
\hline Hipostômio: largura da base & 17,53 \\
\hline Dentes na fila 3 & 52,55 \\
\hline Tarso I: comprimento & Ausente \\
\hline Tarso I: largura & 131,61 \\
\hline Pms: Posteromedial. & 59,56 \\
\hline
\end{tabular}

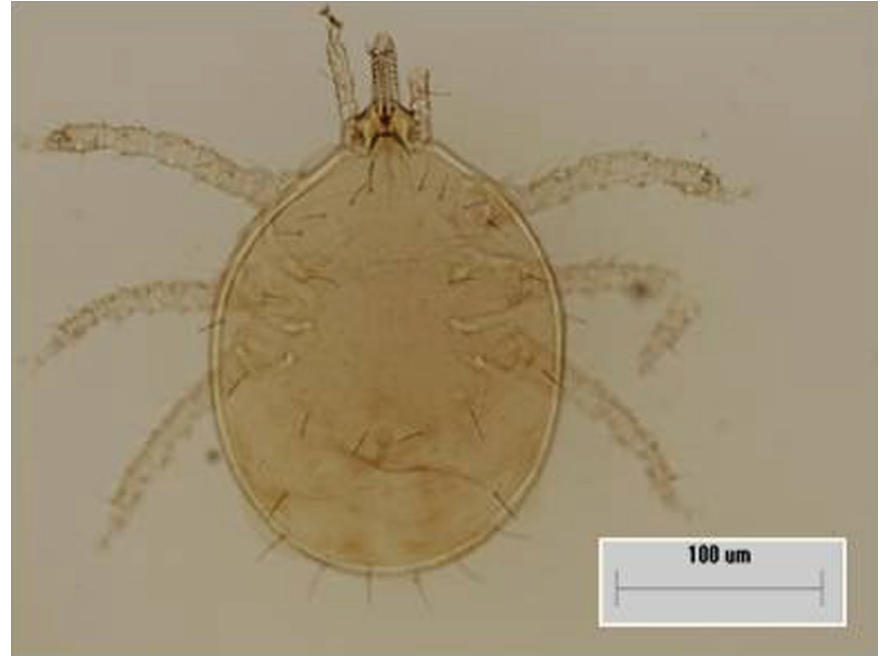

Fig.3. Larva de Ornithodoros mimon. Vista dorsal. Obj.40x.

ca tinha características morfológicas diferentes das encontradas em descrições para carrapatos Ornithodorinae da América. Devido ao estado completamente ingurgitado, a montagem em lâmina deste único exemplar de Ornithodoros permitiu apenas a obtenção das medidas do hipostômio, capitulum (Fig.4) e dos caracteres morfológicos apresentados no Quadro 4. Como o exemplar estudado apresentou alguns fragmentos corporais danificados, somente foi possível a classificação até o gênero, ficando sua identificação restrita a Ornithodoros sp.

Dos 293 carrapatos coletados, 14 espécimes foram submetidos à extração de DNA, seguida da PCR e do sequenciamento para identificação da espécie; 273 espécimes foram depositados no acervo entomológico de ectoparasitos do Laboratório de Parasitologia Animal da UFERSA e 6 na Coleção Nacional de Carrapatos (CNC) da Faculdade de Medicina Veterinária e Zootecnia (FMVZ) da USP, sob os números de tombo: CNC 3064-3068. As 14 larvas de A. auricularium analisadas através da PCR amplificaram fragmentos que comparados com sequências presentes no GenBank apresentaram 99\% (405/407-pb) a 100\% (407-pb) de identidade com A. auricularium do estado de Pernambuco (GenBank KC202817). Já as larvas de 0 . mimon apresentaram identidade de $100 \%$ com 0 . mimon encontrado em Natal, Rio Grande do Norte (GenBank KC677679) e Ipojuca, Pernambuco (GenBank KC677676). As sequências de A. auricularium e $O$. mimon obtidas através da presente pesquisa, foram submetidas no GenBank sob número de acesso KR869153 para $O$. mimon e KR869154-KR869156 para A. auricularium. 
Quadro 3. Morfometria das larvas coletadas de Gracilinanus agilis

\begin{tabular}{|c|c|c|c|c|}
\hline $\begin{array}{c}\text { Larvas } \\
\text { Avaliadas }\end{array}$ & Características & Média \pm D.P & Min-Max & $\mathrm{CV} \%$ \\
\hline 5 & Comprimento com capítulo & $1104,86 \pm 135,25$ & $933,54-1244,39$ & 12,24 \\
\hline 10 & Comprimento sem capítulo & $967,62 \pm 135,94$ & $777,23-1185,88$ & 14,04 \\
\hline 10 & Largura do idiossoma & $800,70 \pm 109,79$ & $688,10-976,86$ & 13,71 \\
\hline 10 & Placa dorsal: comprimento & $165,98 \pm 12,35$ & $142,16-185,08$ & 7,44 \\
\hline 10 & Placa dorsal: largura & $139,85 \pm 6,42$ & $137,11-149,03$ & 4,59 \\
\hline 10 & Cerda dorsal anterolateral: Al1 & $71,01 \pm 5,63$ & $60,51-79,53$ & 7,93 \\
\hline 10 & Cerda dorsal anterolateral: Al2 & $59,71 \pm 3,45$ & $51,90-65,06$ & 5,78 \\
\hline 10 & Cerda dorsal anterolateral: $\mathrm{Al} 3$ & $60,67 \pm 3,39$ & $52,33-65,25$ & 5,59 \\
\hline 10 & Cerda dorsal anterolateral: Al4 & $64,10 \pm 4,40$ & $56,11-69,59$ & 6,87 \\
\hline 10 & Cerda dorsal anterolateral: Al5 & $56,57 \pm 4,16$ & $49,05-60,66$ & 7,36 \\
\hline 10 & Cerda dorsal anterolateral: $\mathrm{Al} 6$ & $64,30 \pm 4,52$ & $56,24-69,55$ & 7,03 \\
\hline 10 & Cerda dorsal anterolateral: $\mathrm{Al} 7$ & $63,53 \pm 3,20$ & $58,92-67,96$ & 5,03 \\
\hline 10 & Cerda dorsal posterolateral: Pl 1 & $77,64 \pm 4,59$ & $72,16-83,33$ & 5,91 \\
\hline 10 & Cerda dorsal posterolateral: Pl 2 & $86,61 \pm 6,26$ & $78,06-94,09$ & 7,23 \\
\hline 10 & Cerda dorsal posterolateral: Pl 3 & $87,53 \pm 8,21$ & $69,03-98,56$ & 9,37 \\
\hline 10 & Cerda dorsal posterolateral: $\mathrm{Pl} 4$ & $83,74 \pm 7,62$ & $71,06-95,39$ & 9,10 \\
\hline 10 & Cerda central: $\mathrm{C} 1$ & $71,39 \pm 6,58$ & $58,43-76,27$ & 9,22 \\
\hline 9 & Cerda central: $\mathrm{C} 2$ & $64,17 \pm 4,04$ & $59,55-71,32$ & 6,30 \\
\hline 9 & Cerda central: C3 & $64,65 \pm 5,16$ & $57,69-73,97$ & 7,98 \\
\hline 10 & Cerda esternal: St1 & $44,33 \pm 2,57$ & $38,99-47,39$ & 5,80 \\
\hline 10 & Cerda esternal: St2 & $42,01 \pm 2,21$ & $37,72-44,62$ & 5,26 \\
\hline 10 & Cerda esternal: St3 & $40,86 \pm 1,86$ & $38,59-44,24$ & 4,55 \\
\hline 10 & Cerda circuanal: Ca1 & $37,03 \pm 2,51$ & $32,66-40,66$ & 6,78 \\
\hline 10 & Cerda circuanal: $\mathrm{Ca} 2$ & $49,87 \pm 2,54$ & $47,25-55,97$ & 5,10 \\
\hline 10 & Cerda circuanal: $\mathrm{Ca} 3$ & $54,66 \pm 4,27$ & $47,67-60,99$ & 7,82 \\
\hline 10 & Cerda Pms & $51,22 \pm 4,97$ & $43,45-58,58$ & 9,70 \\
\hline 10 & Cerda poscoxal: Pc & $34,67 \pm 4,21$ & $27,45-41,13$ & 12,16 \\
\hline 10 & Cerda da placa anal: As & $46,56 \pm 3,78$ & $40,54-51,58$ & 8,11 \\
\hline 9 & Comprimento da base do capítulo até Ph1 & $74,66 \pm 7,49$ & $62,69-77,95$ & 10,03 \\
\hline 6 & $\begin{array}{l}\text { Comprimento da base do capítulo até a } \\
\text { inserção do hipostômio }\end{array}$ & $80,80 \pm 7,80$ & $71,70-93,10$ & 9,66 \\
\hline 4 & Comprimento do capítulo & $235,58 \pm 7,08$ & $229,49-245,82$ & 3,00 \\
\hline 10 & Largura do capítulo & $180,77 \pm 9,59$ & $168,76-193,90$ & 5,31 \\
\hline 9 & Cerda Poshipostomal Ph1 & $9,90 \pm 2,84$ & $5,47-13,49$ & 28,76 \\
\hline 7 & Cerda Pós-hipostomal Ph2 & $16,72 \pm 1,86$ & $15,04-18,49$ & 11,17 \\
\hline 9 & Distância Ph1 - Ph1 & $24,96 \pm 2,63$ & $20,65-28,64$ & 10,57 \\
\hline 7 & Distância Ph2 - Ph2 & $85,41 \pm 4,17$ & $80,75-90,76$ & 4,89 \\
\hline 9 & Comprimento do palpo & $206,21 \pm 3,70$ & $203,12-211,71$ & 1,79 \\
\hline 9 & Comprimento artículo I & $49,83 \pm 1,12$ & $48,41-51,84$ & 2,26 \\
\hline 9 & Comprimento artículo II & $68,61 \pm 6,78$ & $57,07-76,45$ & 9,88 \\
\hline 9 & Comprimento artículo III & $68,62 \pm 2,26$ & $65,13-71,27$ & 3,30 \\
\hline 9 & Comprimento artículo IV & $37,18 \pm 2,02$ & $35,64-40,92$ & 5,44 \\
\hline 2 & $\begin{array}{l}\text { Hipostômio: comprimento até a parte } \\
\text { denteada inferior }\end{array}$ & $148,71 \pm 10,01$ & $141,64-155,79$ & 6,73 \\
\hline 4 & Hipostômio: largura & $50,62 \pm 2,50$ & $48,02-51,03$ & 4,95 \\
\hline 9 & Tarso I: comprimento & $153,93 \pm 5,15$ & $145,83-158,76$ & 3,34 \\
\hline 9 & Tarso I: largura & $57,84 \pm 3,54$ & $53,93-61,96$ & 6,12 \\
\hline
\end{tabular}

Pms: posteromedial; D.P.: Desvio padrão; CV\%: Coeficiente de variação a 95\%; Min-Max: Valores mínimos e máximos.

Quanto a ocorrência de parasitismo por carrapatos, observou-se maior número de exemplares de A. auricularium em $M$. domestica e Thrichomys sp.; e de $O$. mimon, em G. agilis (Quadro 5).

\section{DISCUSSÃO}

Todos os carrapatos coletados, nos pequenos mamíferos capturados, foram encontrados em estágios imaturos, o que justifica a importância destes animais como hospedeiros no ciclo de vida destes ectoparasitos. Para os roedores estudados na presente pesquisa, os ectoparasitos mais ocorrentes foram Amblyomma parvum e Amblyomma auricularium. Infestação por 15 ninfas de $A$. parvum foi obser- vada em um exemplar de Thrichomys sp. e por 31 ninfas de A. auricularium em outro Thrichomys sp. Uma larva de Ornithodoros sp. foi encontrada no membro torácico direito de Thrichomys sp. Larvas de carrapatos deste gênero, descrito originalmente como parasito de Chiroptera, foram observadas em roedores não apenas Echimyidae e gênero Thrichomys sp., mas também em Cricetidae e gênero Wiedomys sp. tanto da Caatinga como de outros biomas (Horta et al. 2011, Landulfo et al. 2013, Sponchiado et al. 2015).

0 parasitismo de Thrichomys sp. por A. parvum foi igualmente registrado por Horta et al. (2011), na região Nordeste do Brasil e no estado de Pernambuco. Nava et al. (2006) e Guglielmone \& Nava (2010) descreveram A. parvum como 


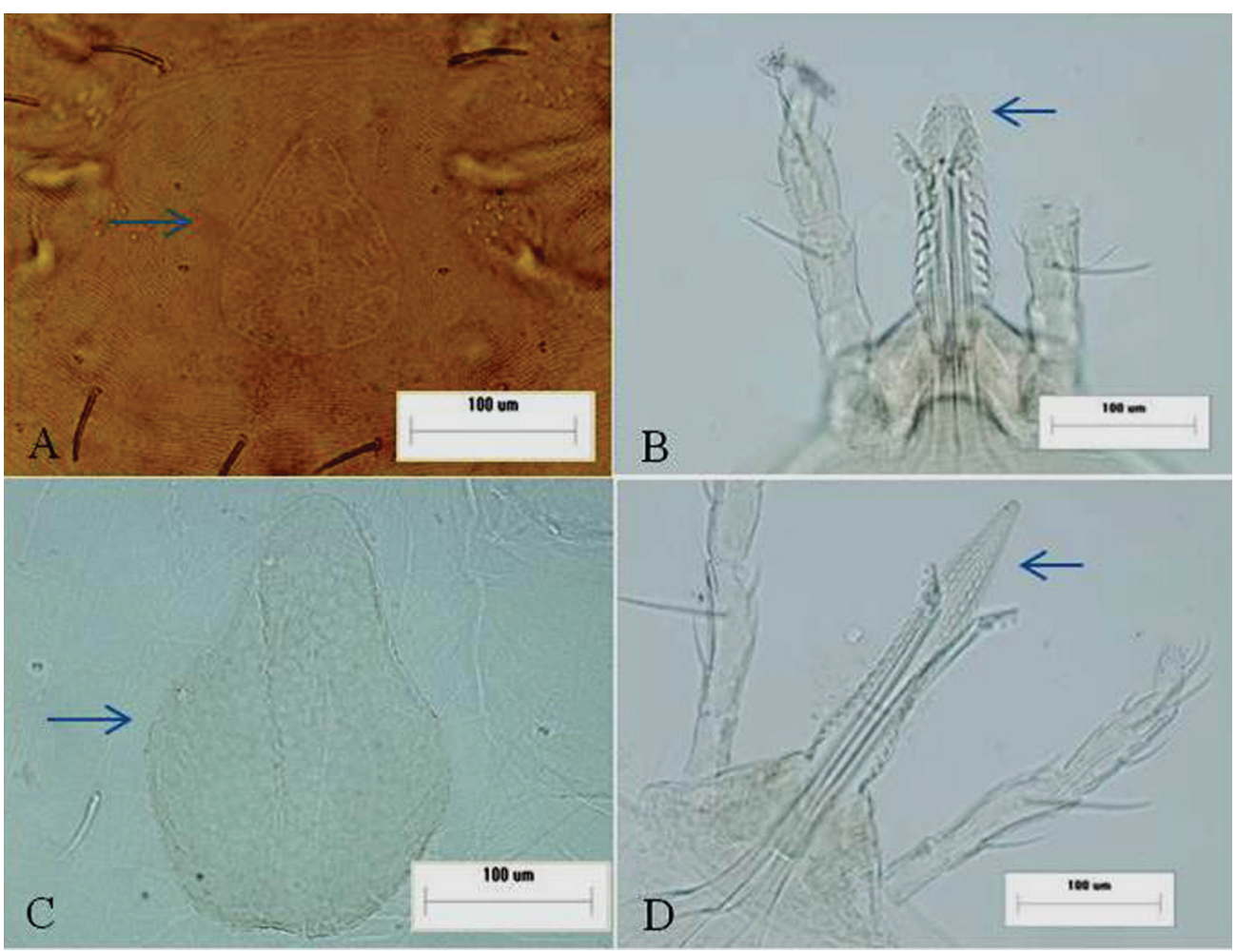

Fig.4. Larva de Ornithodoros mimon. Obj.40x: (A) Formato da placa. (B) Hipostômio (ápice arredondado). Larva de Ornithodoros sp. Obj.40x: (C) Formato da placa. (D) Hipostômio (ápice pontiagudo).

Quadro 4. Morfometria da larva de Ornithodoros sp. coletada de Monodelphis domestica

\begin{tabular}{|c|c|}
\hline Características & Valores $(\mu \mathrm{m})$ \\
\hline Estado de alimentação & Ingurgitada \\
\hline Placa dorsal: comprimento & 310,80 \\
\hline Placa dorsal: largura & 217,92 \\
\hline Comprimento da base do capítulo até Ph1 & 142,38 \\
\hline $\begin{array}{l}\text { Comprimento da base do capítulo } \\
\text { até a inserção do hipostômio }\end{array}$ & 158,38 \\
\hline Comprimento do capítulo & 394,28 \\
\hline Largura do capitulo & 210,49 \\
\hline Cerda Poshipostomal Ph1 & 14,04 \\
\hline Cerda Poshipostomal Ph2 & 25,23 \\
\hline Distância Ph1 - Ph1 & 21,68 \\
\hline Distância Ph2 - Ph2 & 77,30 \\
\hline Comprimento do palpo & 283,22 \\
\hline Comprimento artículo I & 54,15 \\
\hline Comprimento artículo II & 105,78 \\
\hline Comprimento artículo III & 92,16 \\
\hline Comprimento artículo IV & 46,89 \\
\hline Cerdas do artículo I & 0 \\
\hline Cerdas do artículo II & 5 \\
\hline Cerdas do artículo III & 5 \\
\hline Cerdas do artículo IV & 9 \\
\hline Hipostômio: comprimento até a ponta de $\mathrm{Ph} 1$ & 246,44 \\
\hline Hipostômio: comprimento até a parte denteada inferior & 218,96 \\
\hline Hipostômio: largura & 42,74 \\
\hline Hipostômio: Ápice & Pontiagudo \\
\hline Formula dental apical & 2.2 \\
\hline Formula dental medial & $3.3 / 2.2$ \\
\hline Formula dental basal & 2.2 \\
\hline Dentes na fila 1 & $25-26$ \\
\hline Dentes na fila 2 & $22-23$ \\
\hline Dentes na fila 3 & 7 \\
\hline Tarso I: comprimento & 171,64 \\
\hline Tarso I: largura & 66,42 \\
\hline
\end{tabular}

principal espécie que parasita roedores da família Caviidae e gênero Galea sp. A infestação por este ectoparasito em Thrichomys sp. é justificada devido o alto grau de parentesco e semelhanças morfológicas e comportamentais entre espécies de Caviidae e Echimyidae Sulamericanas (Bonvicino et al. 2002, Reis et al. 2006, Bonvicino et al. 2008).

Na Caatinga, adultos de A. auricularium tem sido descritos parasitando principalmente animais de médio porte da família Dasypodidae, os tatus, e a espécie de Carnivora Conepatus semistriatus (Horta et al. 2011, Fonseca et al. 2013). Por outro lado, os principais hospedeiros para larvas e ninfas desta espécie de carrapato têm sido animais de pequeno porte, tais como Galea spixii, Monodelphis domestica e Thrichomys apereoides (Horta et al. 2011).

Além de $A$. parvum e $A$. auricularium, outras espécies de Amblyomma foram descritas parasitando espécies de roedores do gênero Thrichomys sp. na região Nordeste do Brasil, dentre elas Amblyomma ovale (Kock 1844) (Saraiva et al. 2012).

Nos marsupiais estudados, observou-se parasitismo por A. auricularium e Ornithodoros mimon em M. domestica e Gracilinanus agilis. Sponchiado et al. (2015) notificaram O. mimon em G. agilis. Horta et al. (2011), no Nordeste do Brasil, coletaram $A$. auricularium em $M$. domestica. 0 papel de 0 . mimon na transmissão de doenças ainda é desconhecido. No entanto, esse carrapato é muito agressivo para seres humanos, desencadeando reações inflamatórias intensas (Barros-Battesti et al. 2011). Labruna et al. (2014) relataram a infestação por 0 . mimon em Didelphis albiventris (Lund 1840) no Rio Grande do Norte. 0 presente trabalho traz o primeiro registro do parasitismo por 0 . mimon 


\begin{abstract}
Quadro 5. Ocorrência de Carrapatos coletados de pequenos mamíferos capturados na Estação Experimental Rafael Fernandes, Rio Grande do Norte, Brasil, de janeiro de 2014 a fevereiro de 2015
\end{abstract}

\begin{tabular}{lcccc}
\hline Animais capturados (№) & $\begin{array}{c}\text { Animais } \\
\text { positivos }\end{array}$ & $\begin{array}{c}\text { Espécie de } \\
\text { Ectoparasito }\end{array}$ & $\begin{array}{c}\text { № de carrapatos } \\
\text { coletados }\end{array}$ & $\begin{array}{c}\text { Ocorrência (№ de carrapatos/ } \\
\text { № de animais capturados) }\end{array}$ \\
\hline Monodelphis & 11 & A. auricularium & 174 & 12,42 \\
domestica (14) & 1 & Ornithodoros sp. & 1 & 0,07 \\
& 1 & O. mimon & 1 & 0,07 \\
Gracilinanus agilis (38) & 10 & A. auricularium & 11 & 0,28 \\
Wiedomys sp.(5) & 28 & O. mimon & 40 & 1,05 \\
Rattus norvegicus (1) & 5 & Amblyomma sp. & 12 & 2,4 \\
Thrichomys sp. (4) & 1 & Amblyomma sp. & 6 & 6 \\
& 1 & A. auricularium & 31 & 7,75 \\
& 1 & A. parvum & 15 & 3,75 \\
& 1 & Ornithodoros sp. & 1 & 0,25 \\
& 1 & O. mimon & 1 & 0,25
\end{tabular}

(№) = número de animais capturados.

em duas outras espécies de marsupiais; $M$. domestica e $G$. agilis nesse Estado. Segundo Cáceres (2012), estes animais têm hábitos noturnos que podem justificar o seu contato com morcegos, hospedeiros originalmente descritos para essa espécie de Argasidae em ambientes e nichos ecológicos compartilhados entre eles.

Nos espécimes de G. agilis foi observado hábito de autolimpeza constante (grooming) ingerindo, muitas vezes, materiais existentes sobre sua pelagem. Vieira (2014) e Gomes (2008) justificam que estas ações comportamentais explicam o fato desta espécie hospedeira apresentar índices parasitários baixos.

Quanto a classificação das larvas de 0 . mimon por quetotaxia e morfometria, constatou-se que as características analisadas(forma da placa dorsal; fórmula dental apical, medial e basal do hipostômio; ápice do hipostômio; número de cerdas dorsais; dorsolaterais; ventrais; circuanais e do artículo I, II,III e IV dos palpos) foram iguais às descritas por Kohls et al. (1969) e Barros-Battesti et al. (2011).

No que diz respeito a larva de Ornithodoros sp. recuperada em $M$. domestica, pode tratar-se de um morfotipo não descrito, pois a forma do hispostômio, que corresponde a um caráter taxonômico de peso nas espécies do gênero Ornithodoros, não coincide com a morfologia descrita para as espécies da América. Estudos futuros, incluindo análises morfológica e molecular de maior número de larvas deste morfotipo, são necessários para confirmar esta hipótese.

Registra-se a ocorrência das espécies de carrapatos $A$. parvum, $A$. auricularium e $O$. mimon em um ambiente natural, no Rio Grande do Norte, além dos hospedeiros $M$. domestica, G. agilis, Wiedomys sp., Rattus norvegicus e Thrichomys sp. Apesar da grande relevância dos registros fornecidos por este estudo, uma vez que contribuem para o conhecimento de espécies hospedeiras para os Ixodidae e Argasidae nas condições semiáridas deste Estado, trabalhos futuros são necessários para notificação de mais espécies num bioma único do Brasil: a Caatinga.

\section{CONCLUSÕES}

Em Wiedomys sp. e Rattus norvegicus foram identificados carrapatos ixodídeos do gênero Amblyomma sp.; em Monodelphis domestica e Gracilinanus agilis foi constata- do parasitismo por Amblyomma auricularium e, em Thrichomys sp., havia A. auricularium (maior ocorrência) e Amblyomma parvum.

Em M. domestica, G. agilis e Thrichomys sp. foram identificados os carrapatos argasídeos Ornithodoros mimon (maior ocorrência em G. agilis) e Ornithodoros sp.

Agradecimentos.- Ao Programa de Pós-Graduação em Ciência Animal da Universidade Federal Rural do Semi-árido (UFERSA). A UFERSA. Ao Prof. Dr. Michael Hrncir, responsável pela área de trabalho, a Estação Experimental Rafael Fernandes da UFERSA. Ao Dr. Wesley Adson Costa Coelho, pelas análises estatísticas. Ao Laboratório de Doenças Parasitárias e Laboratório de Biologia Molecular aplicado a Sorologia da Universidade de São Paulo, pela realização das análises moleculares. Ao Instituto Brasileiro de Meio Ambiente e dos Recursos Naturais Renováveis (IBAMA), por licença concedida para realização do presente estudo. SML foi financiado pela Beca Chile $\mathrm{N}^{\circ} 72140079$.

\section{REFERÊNCIAS}

Almeida A.J., Freitas M.M.F. \& Talamoni S.A. 2013. Use of space by the Neotropical caviomorph rodent Thrichomys apereoides (Rodentia: Echimyidae). Zoologia 30(1):35-42.

Altschul S.F., Gish W., Miller W., Myers E.W. \& Lipman D.J. 1990. Basic local alinment search tool. J. Molecular Biology 215:403-410.

Aragão H. 1936. Ixodidas brasileiros e de alguns paizes limitrophes. Memórias Inst. Oswaldo Cruz (31):759-843.

Barros-Battesti D.M., Landulfo G.A., Onofrio V.C., Faccini J.L.H., Marcili A., Nieri-Bastos F.A., Venzal J.M. \& Labruna M.B. 2011. Carios mimon (Acari: Argasidae): description of adults and redescription of larva. Exp. Appl. Acarology 54:93-104.

Barros-Battesti D.M., Ramirez D.G., Landulfo G.A., Faccini J.L.H., Dantas-Torres F., Labruna M.B., Venzal J.M. \& Onofrio V.C. 2013. Immature argasid ticks: diagnosis and keys for Neotropical region. Revta Bras. Parasitol. Vet. 22(4):443-456.

Barros-Battesti D.M., Landulfo G.A., Luz H.R., Marcili A., Onofrio V.C. \& Famadas K.M. 2015. Ornithodoros faccinii n. sp. (Acari: Ixodida: Argasidae) parasitizing the frog Thoropa miliaris (Amphibia: Anura: Cycloramphidae) in Brazil. Parasites Vectors 8:268.

Bonvicino C.R., Otazu I.B. \& D'Andrea P.S. 2002. Karyologic evidences of diversification of the genus Thrichomys (Rodentia, Echimyidae). Cytogen. Genome Res. 97:200-204.

Bonvicino C.R., Oliveira J.A. \& D'Andrea P.S. 2008. Guia dos roedores do Brasil, com chaves para gêneros baseadas em caracteres externos. Centro Pan-Americano de Febre Aftosa, OPAS/OMS, Rio de Janeiro. 120p.

Cáceres N.C. 2012. Os marsupiais do Brasil: biologia, ecologia e conservação. UFMS, Campo Grande, MS. 530p.

Dantas-Torres F., Onofrio V.C. \& Barros-Battesti D.M. 2009. The ticks 
(Acari: Ixodida: Argasidae, Ixodidae) of Brazil. Systemat. Appl. Acarology 14:30-46.

Fonseca Z.A.A.S., Pereira J.S., Martins T.F., Coelho W.A.C., Andre W.P.P., Bessa E.N. \& Ahid S.M.M. 2013. Ocorrência de Amblyomma auricularium (Conil, 1878) em tatus-galinha de vida livre (Dasypus novemcinctus) (Xenartra: Dasypodidae) no estado do Ceará, Brasil. Acta Veterinaria Brasilica 7(3):233-236.

FreitaS A.A. 2012. Mamíferos do Nordeste brasileiro: espécies continentais. USEB, Pelotas. 133p.

Gomes M.M.A. 2008. Estrutura das comunidades de artrópodos ectoparasitos em pequenos mamíferos na Ilha Mutum, Porto Rico, Paraná. Dissertação de Mestrado em Ecologia e Conservação, Universidade Federal de Mato Grosso do Sul, Campo Grande, MS. 71p.

Guglielmone A.A. \& Nava S. 2010. Rodents of the subfamily Caviinae (Hystricognathi, Caviidae) as hosts for hard ticks (Acari: Ixodidae). Mastoz Neotropical 17:279-286.

Horta M.C., Nascimento G.F., Martins T.F., Labruna L.B., Machado L.C.P. \& Nicola P.A. 2011. Ticks (Acari: Ixodida) parasitizing free-living wild animals in the Caatinga biome in the State of Pernambuco, northeastern Brazil. Systemat. Appl. Acarology 16:207-211.

Jones E.K. \& Clifford C.M. 1972. The systematics of the subfamily Ornithodorinae (Acarina: Argasidae). V. A revised key to larval Argasidae of the western hemisphere and description of seven new species of Ornithodoros. Annals Entomologic. Soc. America 65:730-740.

Kim H.C., Lee I.Y., Chong S.T., Richards A.L., Gu S.H., Song J.W., Lee J.S. \& Terry A.K. 2010. Serosurveillance of scrub Typhus in small mammals collected from military training sites near the DMZ, Northern Gyeonggi-do, Korea, and analysis of the relative abundance of Chiggers from mammals examined. Korean J. Parasitology 48(3):237-243.

Kohls G.M., Clifford C.M. \& Jones E.K. 1969. The systematics of the subfamily Ornithodorinae (Acarina: Argasidae). IV. Eight new species of Ornithodoros from the Western Hemisphere. Annals Entomologic. Soc. America 62:1035-1043.

Krawczak F.S., Martins T.F., Oliveira C.S., Binder L.C., Costa F.B., Nunes P.H., Gregori F. \& Labruna M.B. 2015. Amblyomma yucumense n. sp. (Acari: Ixodidae), a Parasite of Wild Mammals in Southern Brazil. J. Med. Entomology 52(1):28-37.

Labruna M.B., Jorge R.S.P., Sana D.A., Jacomo A.T.A., Kashivakura C.K., Furtado M.M., Ferro C., Perez S.A., Silveira L., Santos T.S., Marques S.R., Morato R.G., Nava A., Adania C.H., Teixeira R.H.F., Gomes A.A.B., Conforti V.A., Azevedo F.C.C., Prada C.S., Silva J.C.R., Batista A.F., Marvulo M.F.V., Morato R.L.G., Alho C.J.R., Pinter A., Ferreira P.M., Ferreira F. \& Barros-Battesti D.M. 2005. Ticks (Acari: Ixodida) on wild carnivores in Brazil. Exp. Appl. Acarology (36):149-163.

Labruna M.B., Marcili A., Ogrzewalska M., Barros-Battesti D.M., Dantas-Torres F., Fernandes A.A., Leite R.C. \& Venzal J.M. 2014. New Records and Human Parasitism by Ornithodoros mimon (Acari: Argasidae) in Brazil. J. Med. Entomology 51(1):283-287.

Landulfo G.A., Pevidor L.V., Luz H. R., Faccini L.H., Nunes P.H. \& Barros-Battesti D.M. 2013. Description of nymphal instars of Ornithodoros mimon Kohls, Clifford \& Jones, 1969 (Acari: Argasidae). Zootaxa 3710(2):179191.

Mangold A.J., Bargues M.D. \& Mas-Coma S. 1998. Mitochondrial 16S rDNA sequences and phylogenetic relationships of species of Rhipicephalus and other tick genera among Metastriata (Acari: Ixodidae). Parasitol. Res. 84:478-484.

Martins T.F., Onofrio V.C., Barros-Battesti D.M. \& Labruna M.B. 2010. Nymphs of the genus Amblyomma (Acari: Ixodidae) of Brazil: descriptions, redescriptions, and identification key. Ticks Tick-Borne Dis. 1:7599.

Martins T.F., Venzal J.M., Terassini F.A., Costa F.B., Marcili A., Camargo L.M., Barros-Battesti D.M. \& Labruna M.B. 2014. New tick records from the state of Rondônia, western Amazon, Brazil. Exp. Appl. Acarology 62:121-128.

Mastropaolo M., Beltrán-Saavedra L.F. \& Guglielmone A.A. 2014. The ticks
(Acari: Ixodida: Argasidae, Ixodidae) of Bolivia. Ticks Tick-borne Dis. 5:186-194.

Nava S., Mangold A.J. \& Guglielmone A.A. 2006. The natural hosts for larvae and nymphs of Amblyomma neumanni and Amblyomma parvum (Acari: Ixodidae). Exp. Appl. Acarology 40:123-131.

Nava S., Beati L., Labruna M.B., Cáceres A.G., Mangold A.J. \& Guglielmone A.A. 2014. Reassessment of the taxonomic status of Amblyomma cajennense (Fabricius, 1787) with the description of three new species, $A m$ blyomma tonelliae n. sp., Amblyomma interandinum n. sp. and Amblyomma patinoi n. sp., and resurrection of Amblyomma mixtum Koch, 1844 and Amblyomma sculptum Berlese, 1888 (Ixodida: Ixodidae). Ticks Tick Borne Diseases 5:252-276.

Pereira J.S., Melo A.E.C., Sousa F.S.R., Oliveira M.F. \& Ahid S.M.M. 2012. Parasitismo por Gliricola quadrisetosa Ewing, 1924 em Galea spixii Wagler, 1831: redescrição da placa esternal. Acta Veterinaria Brasilica 6(1):45-47.

Reis N.R., Peracchi A.L., Wagner A.P. \& Lima I.P. 2006. Mamíferos do Brasil. Londrina. $437 \mathrm{p}$

Saraiva D.G., Fournier G.F.S.R., Martins T.F., Leal K.P.G., Vieira F.N., Câmara E.M. V.C., Costa C.G., Onofrio V.C., Barros-Battesti D.M., Guglielmone A.A. \& Labruna M.B. 2012. Ticks (Acari: Ixodidae) associated with small terrestrial mammals in the state of Minas Gerais, southeastern Brazil. Exp. Appl. Acarology 58:159-166.

Silva A.F., Pereira D.S., Paiva C.S., Souza R.M. \& Maracajá P.B. 2012. Comportamento defensivo de abelhas africanizadas na Fazenda Experimental, UFERSA, Mossoró/RN, Brasil. Revta Verde de Agroecologia e Desenvolvimento Sustentável 7(5):63-72.

Soares H.S. 2013. Pesquisa de carrapatos, agentes transmitidos por carrapatos e tripanossomatídeos em animais silvestres dos estados do Mato Grosso e Pará. Tese de Doutorado em Epidemiologia Experimental Aplicada às Zoonoses, Faculdade de Medicina Veterinária e Zootecnia, Universidade de São Paulo, SP. 118p.

Soares H.S., Barbieri A.R., Martins T.F., Minervino A.H., Lima J.T.R., Marcili A. \& Labruna M.B. 2015. Ticks and rickettsial infection in the wildlife of two regions of the Brazilian Amazon. Exp. Appl. Acarology 1:1-16

Sponchiado J., Melo G.L., Martins T.F., Krawczak F.S., Labruna M.B. \& Cáceres N.C. 2015. Association patterns of ticks (Acari: Ixodida: Ixodidae, Argasidae) of small mammals in Cerrado fragments, western Brazil. Exp. Appl. Acarology 65:389-401.

Szabó M.P., Labruna M.B., Garcia M.V., Pinter A., Castagnolli K.C., Pacheco R.C., Castro M.B., Veronez V.A., Magalhães G.M., Vogliotti A. \& Duarte J.M. 2009. Ecological aspects of the free-living ticks (Acari: Ixodidae) on animal trails within Atlantic rainforest in south-eastern Brazil. Annals Trop. Med. Parasitol. 103:57-72.

Szabó M.P.J., Niere-Bastos F.A., Spolidorio M.G., Martins T.F., Barbieri A.M. \& Labruna M.B. 2013. In vitro isolation from Amblyomma ovale (Acari: Ixodidae) and ecological aspects of the Atlantic rainforest Rickettsia, the causative agent of a novel spotted fever rickettsiosis in Brazil. Parasitology 140:719-728.

Thompson R.C.A., Kutz S.J. \& Smith A. 2009. Parasite zoonoses and wildlife: emerging issues. International journal of environmental research and Wildlife: emerging issues. Int. J. Environ. Res. Publ. Health 6:678-693.

Venzal J.M., Estrada-Peña A., Mangold A.J., González-Acuña D. \& Guglielmone A.A. 2008. The Ornithodoros (Alectorobius) talaje species group (Acari: Ixodida: Argasidae): description of Ornithodoros (Alectorobius) rioplatensis n. sp. from southern South America. J. Med. Entomol. 45:832-840

Vieira J.P.F.A. 2014. Comunidades d Artrópodes ectoparasitos em pequenos mamíferos de um remanescente de Cerrado em região urbana de Campo Grande, Mato Grosso do Sul. Dissertação de Mestrado em Biologia Animal, Universidade Federal de Mato Grosso do Sul, Campo Grande, MS. 45p.

Wolf R.F., Aragona M., Muñoz-Leal S., Pinto L.B., Melo A.L.T., Braga I.A., Costa J.S., Martins T.F., Marcili A., Pacheco R.C., Labruna M.B. \& Aguiar D.M. 2016. Novel Babesia and Hepatozoon agents infecting non-volant small mammals in the Brazilian Pantanal, with the first record of the ticks Ornithodoros guaporensis in Brazil. Ticks Tick-Borne Dis. 7(3):449-456. 